\title{
Selection of Leaf Orientation Insensitive Bands for Yellow Rust Detection
}

\author{
Lin Yuan ${ }^{1,2}$, Jingcheng Zhang ${ }^{1}$, Jinling Zhao ${ }^{1}$, Shuhong Cai $^{3}$, and Jihua Wang ${ }^{1,2, *}$ \\ ${ }^{1}$ Beijing Research Center for Information Technology in Agriculture, Beijing, 100097, China \\ ${ }^{2}$ Institute of Agriculture Remote Sensing and Information System Application, \\ Zhejiang University, Hangzhou, 310029, China \\ ${ }^{3}$ Hebei Agricultural Technology Extension Station, Shijiazhuang, 050000, China
}

\begin{abstract}
The disease detection by means of hyperspectral reflectance is influenced by the spectral differences between frontside (adaxial surface) and backside (abaxial surface) of a leaf inevitably. Taking yellow rust as an example, this study investigated the spectral differences between frontside and backside of healthy and diseased wheat leaves at grain filling stage using large size samples. We attempted to detect yellow rust with reflectance that was sensitive to the disease and insensitive to the orientation of leaves. The spectral difference between frontside and backside of leaves was analyzed by band ratioing and a pairwise t-test. The bands that were insensitive to the orientation of leaves were identified with a thresholding method. Then, with the aid of an independent t-test analysis, we recognized the bands that were sensitive to the disease. The overlapped bands were applied for developing models that quantifying disease severity by fisher linear discrimination analysis (FLDA). The results suggested that the bands within 606-697nm and 740-1000nm were suitable for disease detection yet insensitive to the orientation of leaves. Based on these bands, the model accuracies reached $71 \%$ for FLDA. These bands can be used as a basis for further selection of appropriate bands to detect yellow rust at canopy level.
\end{abstract}

Keywords: yellow rust, winter wheat, frontside and backside, fisher linear discrimination analysis (FLDA).

\section{Introduction}

Winter wheat (Triticum aestivum L.) is one of the most important crops in China. Yellow rust disease which is induced by Puccinia striiformis f. sp. Tritici, is one of the most destructive diseases that has a severe impact on both yield and grain quality of winter wheat [1-2]. Currently, scouting by human being is the widely used method for crop diseases inspection, which is not only time consuming and labor intensive, but also expensive and subjective. As the only way in obtaining the disease distribution information spatially, remote sensing is a promising alternative to traditional methods in disease monitoring [3]. A number of studies were conducted in understanding the

\footnotetext{
* Corresponding author.
} 
spectral characteristics of yellow rust [4-7]. The wavelengths at $446-725 \mathrm{~nm}$ and 1380-1600 nm were identified as sensitive bands for disease detection [8]; Based on the spectral reflectance at $680 \mathrm{~nm}, 725 \mathrm{~nm}$ and $750 \mathrm{~nm}$, Moshou et al. [9-10] successfully distinguished infected wheat leaves from healthy ones at an early infection stage. It should be noted that in most of the studies that were conducted at leaf level, the leaf spectra were measured only for the frontside of leaves. However, in fact, due to the curling of leaves, the frontside and backside of leaves are presenting in a mixed pattern in the nadir view at canopy level. The spectral difference between the frontside and backside of leaves may complicate the spectral response at canopy level and even lead to the failure of disease detection. Therefore, it is necessary to understand the spectral difference between frontside and backside of healthy and diseased leaves, and search for those bands that were sensitive to the disease yet insensitive to the orientation of leaves. However, the spectral difference of frontside and backside of leaves is rarely reported for healthy or diseased wheat plants. Our objectives were: (1) to analyze the spectral difference between frontside and backside of leaves at grain filling stage, and to identify bands that were insensitive to the orientation of leaves, (2) to identify those bands that were sensitive to the occurrence of disease, and (3) to develop model for disease detection based on those identified bands.

\section{Materials and Methods}

\subsection{Study Area and Yellow Rust Inoculation}

The experiment was conducted at Beijing Xiaotangshan Precision Agriculture Experimental Base, in Changping district, Beijing $\left(40^{\circ} 10.6^{\prime} \mathrm{N}, 116^{\circ} 26.3^{\prime}\right.$ E) on the 2010-2011 growing season. The cultivar of winter wheat was 'Jingdong 9843', which was moderately susceptible to yellow rust. The soil at this site is a silt-clay loam. The average topsoil nutrient status $(0-0.30 \mathrm{~m}$ depth $)$ was as follows: organic matter $1.42-1.48 \%$, total nitrogen $0.08-0.10 \%$, alkali-hydrolysis nitrogen $58.6-68.0 \mathrm{mg} \mathrm{kg}^{-1}$, available phosphorus $20.1-55.4 \mathrm{mg} \mathrm{kg}^{-1}$, and rapidly available potassium 117.6-129.1 $\mathrm{mg} \mathrm{kg}^{-1}$. The experimental field received $200 \mathrm{~kg}$ ha-1 nitrogen and $450 \mathrm{~m} 3 \mathrm{ha}^{-1}$ water, which was a recommended rate for this cultivar. Spray method was used in inoculating yellow rust spores to wheat plants. Two different concentrations of spore solutions were applied to generate various infection levels.

\subsection{Inspection of Disease Severity}

The disease severity of each sample was determined by visual estimation of the cover percentage of pustules on the leaf [11-12]. To minimize the error induced by investigator, the diseased leaves were inspected by one investigator. In this study, the disease severity of leaves was determined on the basis of the proportion of infected region on the leaf $(0-100 \%)$. Apart from the healthy leaves, all diseased leaves were 
grouped into 3 severity classes: slight for proportion within 5-25\%, moderate for proportion within $25-60 \%$, and heavy for proportion over $60 \%$. Since those leaves with an infected proportion less than $5 \%$ were actually difficult to be visually separated from healthy ones, they are classified as healthy leaves.

\subsection{Leaf Sampling and Spectral Measurement}

The leaf sampling and measurement were conducted on May 23 (grain filling stage) in the year of 2011. The spectra of frontside and backside were measured for each leaf. The leaves were cut from the plants in the fields with scissors. After that, the samples were immediately packed with ice bags and transported to a nearby indoor laboratory to be measured. A total of 91 leaf samples were measured, including 26 healthy leaves and 65 diseased leaves. All samples were separated for model calibration (60\%) and validation $(40 \%)$ randomly.

Leaf spectral measurements were made by a FieldSpec® UV/VNIR spectroradiometer (ASD Inc., Boulder, Colorado, USA) over the 350-2500 nm wavelengths, coupling with an ASD Leaf Clip. The spectrum of a white Spectralon reference panel (99\% reflectance) was measured once for every 10 leaf measurements. Ten readings were recorded and then averaged to obtain a spectral measurement for each leaf.

\subsection{Comparison between Frontside and Backside of Leaves}

Apart from comparing the frontside and backside of leaves by their original spectra, the band ratioing and pairwise t-test were adopted for the spectral comparison. The band ratioing was used to emphasize the spectral difference between frontside and backside of leaves, whereas the pairwise t-test provided a more explicit way to quantify the spectral differences between two sides.

\subsection{Band Selection and Disease Severity Estimation}

The significance level of spectral differences between healthy and diseased leaves was quantified by an independent t-test analysis for each band, whereas the significance level of spectral differences between the frontside and backside of leaves was quantified by a pairwise t-test. To eliminate the impact from the leaf orientation, only those bands that were sensitive to disease yet insensitive to the orientation of leaves were chosen. Such band selection was facilitated by an overlapping procedure based on the results from the independent t-test and pairwise t-test as illustrated above. The efficiency of these overlapped bands was then tested by comparing the disease severity estimates generated by a Fisher linear discriminate analysis (FLDA) with the measured value [13]. Five measures, overall accuracy (OAA), average accuracy (AA), producer's accuracy, user's accuracy, and kappa coefficient were calculated from confusion matrix to evaluate the accuracies of the discriminate model. 


\section{$3 \quad$ Results}

\subsection{Spectral Differences between Frontside and Backside of Leaves}

The curves of raw reflectance, band ratios between frontside and backside, and corresponding $p$-values of pairwise t-test were summarized in Fig. 1. From the ratio curves, it is obvious that the spectral differences between the frontside and backside of healthy leaves showed a similar pattern with diseased leaves. The spectral reflectance of backside was higher than the frontside in most of the bands, except for the bands at $646-690 \mathrm{~nm}$. For the results of pairwise t-test, a threshold of $p$-value $<0.05$ was used to identify those insensitive bands to the orientation of leaves. The identified bands differed greatly in their positions between healthy and diseased samples. The bands within 741-923 nm were found to be orientation insensitive for healthy leaves, whereas the bands within $606-697 \mathrm{~nm}$ and $740-1000 \mathrm{~nm}$ were identified as orientation insensitive for diseased leaves. It should be noted that most of these bands were located at some specific positions that were responsible for the absorption of chlorophyll and water, and cellular structure.



a

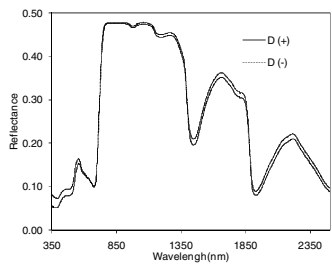

b

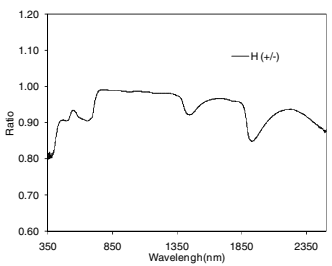

C

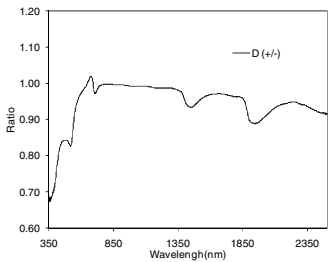

d

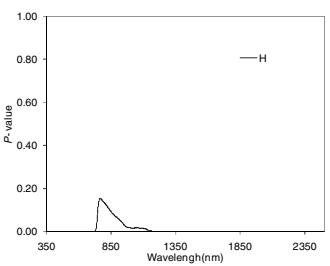

$\mathrm{e}$

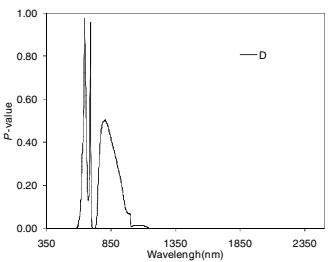

$\mathrm{f}$

Fig. 1. Curves of original reflectance, band ratios between frontside and backside and their corresponding $p$-values based on pairwise t-test

$(a, b)$ curves of original reflectance; $(c, d)$ curves of band ratio; $(e, f)$ curves of $p$-value. " $H$ " indicates the healthy leaf samples; " $D$ " indicates the diseased leaf samples. (+) indicates the frontside of leaves, (-) indicates the backside of leaves.

\subsection{Selection of Effective Wavelengths and Disease Severity Determination}

As shown in Fig. 2, the spectral difference for most bands reached the significant level except for several bands at the beginning of the spectrum. This phenomenon indicated 
that the disease can induce a clear spectral response within a wide spectral region from visible to shortwave near infrared regions. Combining with the bands that were selected in section 3.1, it is possible to generate bands that were sensitive the disease yet insensitive to leaf orientation through an overlapping procedure. As shown in Fig.3, only the bands at 606-697 $\mathrm{nm}$ and 740-1000 $\mathrm{nm}$ were retained. These overlapped bands are theoretically suitable for disease detection since they are able to suppress the disturbance from the leaf orientation when monitoring yellow rust disease. Based on these bands, the FLDA model was established based on the calibration dataset (Table 1). The model accuracies were satisfactory in general, with OAA of 0.71 and kappa coefficient of 0.61 for calibration samples, and OAA of 0.70 and kappa coefficient of 0.59 for validation samples (Table 1).

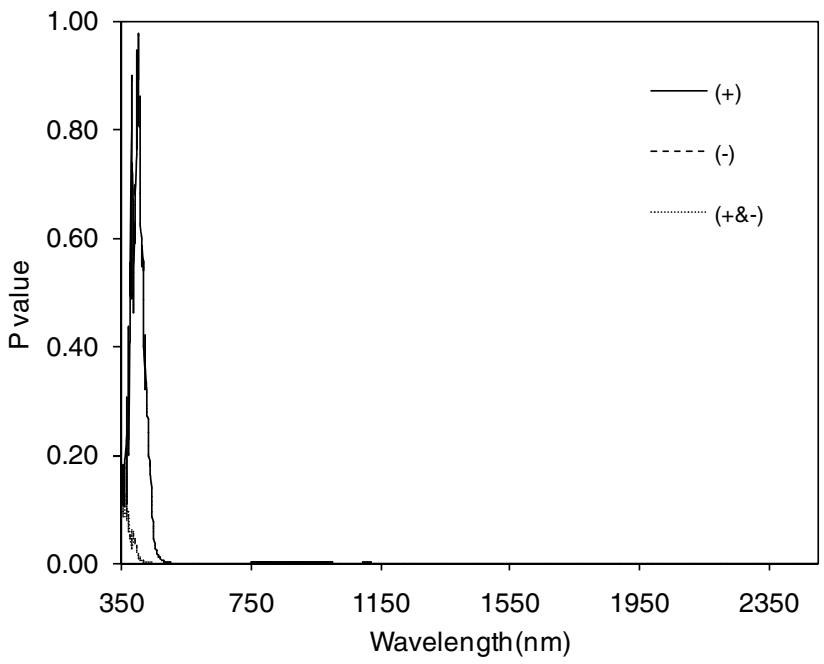

Fig. 2. Curves of the $p$-values of independent t-test between disease severity and band reflectance (+) indicates the frontside of leaves, (-) indicates the backside of leaves, $(+\&-)$ indicates the pooled of both

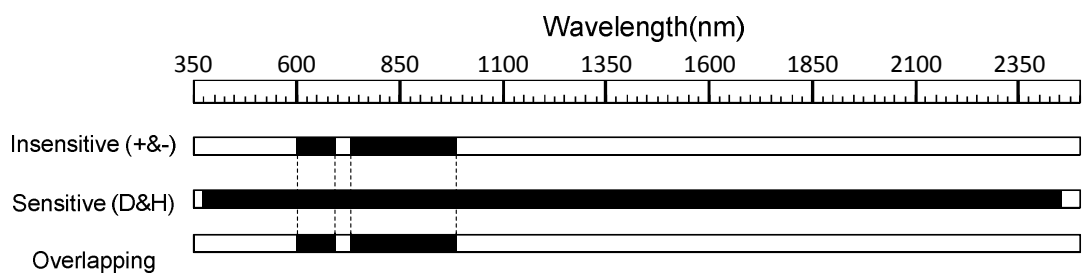

Fig. 3. Overlapping band selection

"Insensitive(+\&-)" represent bands that are insensitive to the orientation; whereas "Sensitive(D\&H)" represent bands that are sensitive to the yellow rust 
Table 1. Confusion matrices created based on the FLDA models

\begin{tabular}{|c|c|c|c|c|c|c|c|c|c|c|}
\hline & & \multicolumn{4}{|c|}{ Reference } & \multirow[b]{2}{*}{ Sum } & \multirow[b]{2}{*}{ U.'s a. (\%) } & \multirow[b]{2}{*}{ OAA } & \multirow[b]{2}{*}{ AA } & \multirow[b]{2}{*}{ Kappa } \\
\hline & & Healthy & Slight & Moderat & Heavy & & & & & \\
\hline \multirow{6}{*}{ Calibration } & Healthy & 25 & 4 & 1 & 0 & 30 & 83.33 & 0.71 & 0.73 & 0.61 \\
\hline & Slight & 5 & 23 & 5 & 0 & 33 & 69.70 & & & \\
\hline & Moderate & 2 & 5 & 12 & 1 & 20 & 60.00 & & & \\
\hline & Heavy & 0 & 2 & 6 & 17 & 25 & 68.00 & & & \\
\hline & Sum & 32 & 34 & 24 & 18 & 108 & & & & \\
\hline & P.'s a. (\%) & 78.13 & 67.65 & 50.00 & 94.44 & & & & & \\
\hline \multirow{6}{*}{ Validation } & Healthy & 16 & 4 & 0 & 0 & 20 & 80.00 & 0.70 & 0.73 & 0.60 \\
\hline & Slight & 2 & 16 & 4 & 0 & 22 & 72.73 & & & \\
\hline & Moderate & 2 & 4 & 8 & 0 & 14 & 57.14 & & & \\
\hline & Heavy & 0 & 0 & 6 & 12 & 18 & 66.67 & & & \\
\hline & Sum & 20 & 24 & 18 & 12 & 74 & & & & \\
\hline & P.'s a. (\%) & 80.00 & 66.67 & 44.44 & 100 & & & & & \\
\hline
\end{tabular}

OAA is overall accuracy; AA is average accuracy; P.'s a. represents producer's accuracy; U.'s a. represents user's accuracy.

\section{Discussion and Conclusion}

In this study, based on the leaf spectra data with a large sample size, the reflectance of frontside and backside were found to be with significant difference at several bands. This finding was in good agreement with Zhou and Wang (2002)'s study, which compared the reflectance of frontside and backside of rice leaves under different nitrogen treatments [14]. Differences in surface reflectance were attributed to the dorsiventral morphology of wheat leaves. This might related with the larger portion of sclerenchyma, vascular bundle and bulliform cells on the upper leaf side of graminaceae [15-16]. The absolute difference of reflectance between the two surfaces was about $4 \%$ for the healthy leaves, and $3 \%$ for the diseased leaves, which means that the occurrence of disease diminished the spectral difference between frontside and backside of leaves. It was found that the reflectance of yellow rust pustules was higher than healthy positions at visible spectral region [17]. The appearance of those pustules at both surfaces for diseased leaves may whiten the spectral difference between the frontside and backside for healthy positions, which thereby lead to a reduction of spectral difference for those diseased leaves. The spectral difference between frontside and backside was relatively weak, comparing with the spectral signals that were induced by disease.

With a spectral overlapping procedure, the spectral reflectance at $606-697 \mathrm{~nm}$ and 740-1000 nm bands were selected for the detection of yellow rust since it can eliminate the impact from the orientation of leaves. However, it should be noted that the present study was conducted at leaf scale, which did not consider more complicated situations that might existed at canopy scale. For example some leaves may present as partially frontside and partially backside. To account for this situation, the spectral imaging techniques and some radiometric transferring models, such as PROSPECT+SAIL should be incorporated for mechanism study and model development.

Acknowledgments. This work was subsidized by National Key Technology R\&D Program (2012BAH29B02), National Natural Science Foundation of China (41071276, 41101395), Beijing Natural Science Foundation (4122032). The authors are grateful to Mr. Weiguo Li, and Mrs. Hong Chang for data collection. 


\section{References}

1. Li, G.B., Zeng, S.M., Li, Z.Q.: Integrated Management of Wheat Pests, pp. 185-186. Press of Agriculture Science and Technology of China, Beijing (1989) (in Chinese)

2. Strange, R.N., Scott, P.R.: Plant Disease: A threat to global food security. Annual Reviews Phytopathol 43, 83-116 (2005)

3. Muhammed, H.H.: Hyperspectral crop reflectance data for characterizing and estimating fungal disease severity in wheat. Biosystems Engineering 91, 9-20 (2005)

4. Bravo, C., Moshou, D., West, J.S., McCartney, A., Ramon, H.: Early disease detection in wheat fields using spectral reflectance. Biosystems Engineering 84, 137-145 (2003)

5. Huang, W.J., Huang, M.Y., Liu, L.Y., Wang, H., Zhao, C.J., Wang, J.D.: Inversion of the severity of winter wheat yellow rust using proper hyperspectral index. Transactions of the CSAE 21, 97-103 (2005)

6. Huang, W.J., David, W.L., Niu, Z., Zhang, Y.J., Liu, L.Y., Wang, J.H.: Identification of yellow rust in wheat using in-situ spectral reflectance measurements and airborne hyperspectral imaging. Precision Agriculture 8, 187-197 (2007)

7. Zhang, J.C., Huang, W.J., Li, J.Y., Yang, G.J., Luo, J.H., Gu, X.H., Wang, J.H.: Development, evaluation and application of a spectral knowledge base to detect yellow rust in winter wheat. Precision Agriculture 12, 716-731 (2011)

8. Huang, M.Y., Huang, W.J., Liu, L.Y., Huang, Y.D., Wang, J.H., Zhao, C.J., Wan, A.M.: Spectral reflectance feature of winter wheat single leaf infected with stripe rust and severity level inversion. Transactions of the CSAE 20, 176-180 (2004); (in Chinese with English abstract)

9. Moshou, D., Bravo, C., West, J., Wahlen, S., McCartney, A., Ramon, H.: Automatic detection of 'yellow rust' in wheat using reflectance measurements and neural networks. Computers and Electronics in Agriculture 44, 173-188 (2004)

10. Moshou, D., Bravo, C., Oberti, R., West, J., Bodria, A., McCartney, A., Ramon, H.: Plant disease detection based on data fusion of hyper-spectral and multi-spectral fluorescence imaging using Kohonen maps. Real-Time Imaging 11, 75-83 (2005)

11. Graeff, S., Link, J., Claupein, W.: Identification of powdery mildew (Erysiphe graminis sp. tritici) and take-all disease (Gaeumannomyces graminis sp. tritici) in wheat (Triticumaestivum L.) by means of leaf reflectance measurements. Central European Journal of Biology 1, 275-288 (2006)

12. Luedeling, E., Hale, A., Zhang, M.H., Bentley, W.J., Dharmasri, L.C.: Remote sensing of spider mite damage in California peach orchards. International Journal of Applied Earth Observation and Geoinformation 11, 244-255 (2009)

13. McLachlan, G.J.: Discriminant analysis and statistical pattern recognition. Wiley Interscience (2004)

14. Zhou, Q.F., Wang, J.H.: Comparison of adaxial and abaxial surface reflectance under different nitrogen level. Transactions of the CSAE 18, 34-39 (2002)

15. Takahashi, K., Mineuchi, K., Nakamura, T., Koizumi, M., Kano, H.: A system for imaging transverse distribution of scattered light and chlorophyll fluorescence in intact rice leaves. Plant Cell \& Environment 17, 105-110 (1994)

16. Kirby, E.J.M.: Botany of the wheat plant. In: Bread Wheat, Improvement and Production (Curtis B C; Rajaram S; Gomez Macpherson Heds). FAO Plant Production Series No. 30, Rome (2002)

17. Devadas, R., Lamb, D.W., Simpfendorfer, S., Backhouse, D.: Evaluating ten spectral vegetation indices for identifying rust infection in individual wheat leaves. Precision Agriculture 10, 459-470 (2009) 